\title{
Time in Range, as a Novel Metric of Glycemic Control, Is Reversely Associated with Presence of Diabetic Cardiovascular Autonomic Neuropathy Independent of HbA1c in Chinese Type 2 Diabetes
}

\author{
Qingyu Guo $\mathbb{D}^{1},{ }^{1}$ Pu Zang $\left(\mathbb{D},{ }^{1}\right.$ Shaoying Xu $\mathbb{D}^{2},{ }^{2}$ Wenjing Song $\mathbb{D}^{\circ},{ }^{3}$ Zhen Zhang $\mathbb{D}^{4}{ }^{4}$ \\ Chunyan Liu $\mathbb{D}^{5}$, Zhanhong Guo $\mathbb{D}^{6}$, Jing Chen $\mathbb{D}^{2},{ }^{2}$ Bin Lu $\mathbb{D},{ }^{1}$ Ping Gu $\mathbb{D}^{1}$, \\ and Jiaqing Shao $\mathbb{D}^{1}$ \\ ${ }^{1}$ Jinling Hosp Dept Endocrinology, Nanjing Univ, Sch Med, Nanjing, China \\ ${ }^{2}$ Jinling Hosp Dept Endocrinology, Southeast Univ, Sch Med, Nanjing, China \\ ${ }^{3}$ Shanghai Sixth People's Hospital East, Shanghai, China \\ ${ }^{4}$ The Seventh Affiliated Hospital, Sun Yat-sen University, Shenzhen, China \\ ${ }^{5}$ Affiliated Hospital of Jiangnan University, Wuxi, China \\ ${ }^{6}$ Jinling Hosp Dept Endocrinology, Nanjing Med Univ, Nanjing, China
}

Correspondence should be addressed to Ping Gu; ping19762000@163.com and Jiaqing Shao; shaojiaqing@nju.edu.cn

Received 6 November 2019; Revised 7 January 2020; Accepted 25 January 2020; Published 6 February 2020

Academic Editor: Yoshifumi Saisho

Copyright ( 2020 Qingyu Guo et al. This is an open access article distributed under the Creative Commons Attribution License, which permits unrestricted use, distribution, and reproduction in any medium, provided the original work is properly cited.

Objective. The objective of this study is to investigate the relationship between time in range (TIR), a new metric of continuous glucose monitoring (CGM) and cardiovascular autonomic neuropathy (CAN) in individuals with type 2 diabetes mellitus (T2DM). Methods. A total of 349 individuals with T2DM were enrolled in this study. Evaluating by the standard cardiac autonomic reflex tests (CARTs), there were 228 diabetic individuals without cardiovascular autonomic neuropathy (without confirmed CAN) including absent CAN ( $n=83$ cases) and early CAN ( $n=145$ cases) and 121 diabetic individuals complicated with cardiovascular autonomic neuropathy (CAN) including definite CAN ( $n=109$ cases) and severe CAN ( $n=12$ cases). All patients underwent 3-day CGM. TIR is defined as the time percent during a 24-hour period when the glucose is in the range of 3.9-10 mmol/L. The Spearman analysis was used to analyze the correlation between TIR and CART parameters, total CAN score. The logistic regression was applied to analyze the relationship between TIR and CAN by adjusting for the age, duration of diabetes, sex, lipid situation, serum creatinine, body mass index, blood pressure, HbAlc (\%), and other glycemic variability (GV) metrics. Results. The total presence of CAN was 34.67\% (definite CAN 31.23\% and severe CAN 3.44\%). Patients with more severe CAN had lower TIR $(P<0.001)$. With increasing quartiles of TIR, the presence of CAN by severity declined $(P<0.05)$. TIR is inversely correlated with total score of CAN $(P<0.001)$ and positively associated with heart rate variation during the lying to standing, Valsalva maneuver, and deep breathing $(P<0.05)$. The logistic regression found a robust association between TIR and CAN independent of HbAlc and GV metrics. Conclusion. TIR is associated with the presence of CAN independent of HbAlc and GV metrics in Chinese type 2 diabetes.

\section{Introduction}

With the rapidly developing technology and increasing use of continuous glucose monitoring (CGM), CGM has been the optimal method to get information on glycemic profile throughout the day. CGM with high accuracy can reflect an individual's glycemic status including hyperglycemia, hypoglycemia, glycemic variability (GV), and daily patterns of glycemia, which provides indication for the treatment of diabetes mellitus [1]. The capability to grade blood glucose levels into different ranges has been convenient likely due, in great part, to increasing use of CGM. The emerging 
metric time in range (TIR) typically refers to the time percent during a 24-hour period when the glucose is in the range of 3.9-10 $\mathrm{mmol} / \mathrm{L}$. A recent international consensus conference proposed that TIR should be the key CGM-derived metric describing short-time glycemic control [2]. Recently, a study stated that TIR is the most paramount indicator for diabetic patients in choosing treatment methods [3]. What is more, other study indicated an association between a high percent of TIR with improvement of hypoglycemic awareness in type one diabetic individuals after intrahepatic islet transplantation [4].

Cardiovascular autonomic neuropathy (CAN) is one of the most shared complications of diabetes, with autonomic imbalance including sympathetic system hyperactivity and parasympathetic system hypoactivity [5]. The ACCORD study confirmed that CAN increased the mortality of type 2 diabetes mellitus (T2DM), the mortality rate of diabetic CAN was 1.55 to 2.14 times than non-CAN, and CAN was related to ascending all-cause and cardiovascular disease mortality [6]. Persistent hyperglycemia and hypoglycemia damage nerves through oxidative stress, eventually leading to the occurrence of autonomic neuropathy [7]. At present, several studies have reported associations of GV metrics assessed by CGM with autonomic neuropathy [8]. What is more, recent researches found an association of TIR with diabetic retinopathy (DR) and diabetic nephropathy (DN) in type 1 diabetes mellitus (T1DM) [9], but the correlation between TIR and CAN has not been studied to date.

The goal of this work was to research whether TIR measured by CGM is connected to the presence and severity of CAN.

\section{Research Design and Methods}

2.1. Participants. A total of 349 individuals (age $\geq 18$ years) with T2DM admitted to the endocrinology department of the Jinling Hospital, Nanjing University, from April 2018 to August 2019 were recruited, all of whom conformed to the 1999 WHO diagnostic criteria for type 2 diabetes. Evaluating by the standard cardiac autonomic reflex tests (CARTs), there were 228 diabetic individuals without CAN (without confirmed CAN) including absent CAN ( $n=83$ cases) and early CAN ( $n=145$ cases) and 121 diabetic individuals complicated with CAN including definite CAN ( $n=109$ cases) and severe CAN ( $n=12$ cases). The mean age of all individuals was $53.11 \pm 12.86$ years. Exclusion criteria included (1) patients with acute complications of diabetes, acute stress such as severe infection, trauma and surgery, severe cardiovascular and cerebrovascular diseases, severe respiratory disease, malignant disease, and pregnancy; (2) patients with definite hepatic or gallbladder disease; (3) patients with narcotic and psychotropic drugs, and a recent history of alcoholism. The study was supported by the local ethics committee and all individuals gave informed consent.

2.2. Clinical and Biochemical Measurements. General clinical information and physical examination such as age, gender, diabetes duration, history of smoking, hypertension, diabetic retinopathy, and the treatment of diabetes were recorded.
Height, weight, systolic blood pressure (SBP), and diastolic blood pressure (DBP) were measured. Body mass index (BMI) was computed. Biochemical measurements such as blood and urine samples were tested after overnight fasting. Hemoglobin A1C (HbA1c), triglyceride (TG), total cholesterol (TC), high-density lipoprotein (HDL), low-density lipoprotein (LDL), fasting blood glucose (FBG), fasting C-peptide, blood urea nitrogen (BUN), and serum creatinine (SCr) were detected.

2.3. CGM Parameters. The continuous glucose detection system of MiniMed Company and Meiqi Company was used to monitor glucose for 72 hours continuously, and capillary blood glucose at least four times every day was used to update the monitor according to procedures. Intensive activities were prohibited in the course of glucose monitoring. Based on the original blood glucose data recorded by this system, a number of metrics concerning mean blood glucose (MBG) and glycemic variability (GV), including standard deviation (SD), mean amplitude of glucose excursions (MAGE), coefficient of variation $(\mathrm{CV})$, largest amplitude of plasma glucose excursions (LAGE), average daily risk range (ADDR), and $M$ value were tested using the EasyGV Version 9.0R2 published by Oxford University. TIR was assessed as the time percent during a 24-hour period when the glucose is in the range of 3.9-10 $\mathrm{mmol} / \mathrm{L}$.

2.4. Cardiac Autonomic Function Testing. Standard CARTs were performed on all of the enrolled patients. All operations were performed by the only medical staff. Smoking, drinking, and eating were prohibited and drugs like antihistamines and beta-blockers were not allowed twelve hours before the examination. The CARTs were performed using a standard protocol [10]: [1] Valsalva R-R ratio: determined the maximum $\mathrm{R}-\mathrm{R}$ interval and the minimum $\mathrm{R}-\mathrm{R}$ interval after Valsalva maneuver. Valsalva R-R ratio greater than or equal to 1.21 was normal, $1.11 \sim 1.20$ was borderline, and less than or equal to 1.1 was abnormal. (2) Heart rate (HR) response to deep breathing: measured the maximum and minimum $\mathrm{R}-\mathrm{R}$ interval during each respiratory cycle and turned into heart rate. Determined the mean of the difference between the maximum heart rate and the minimum heart rate in each of the six deep breathing cycles within 1 minute. The difference value of HR responses to deep breathing greater than or equal to 15 was normal, 11 14 was borderline, and less than or equal to 10 was abnormal. [3] HR response to standing: measured the longest R-R interval in $28 \sim 32$ heart beats and the shortest R-R interval in 13 17 heart beats, converted to heart rate, during patients from lying to standing. The ratio of heart rate between standing and lying position greater than or equal to 1.04 was normal, $1.01 \sim 1.03$ was borderline, and less than or equal to 1.0 was abnormal. [4] Systolic blood pressure response to standing: The difference of systolic blood pressure between lying down and after standing up for 2 minutes was measured. Systolic difference value less than or equal to $10 \mathrm{mmHg}(1 \mathrm{mmHg}=0.133 \mathrm{kpa})$ was normal, $10 \sim 20 \mathrm{mmHg}$ was borderline, and greater than or equal to $20 \mathrm{mmHg}$ was abnormal. Each test had a score of $0,0.5$, or, 1 if it was in a condition of normal, borderline, 
or abnormal range, respectively. Therefore, the minimum and maximum score were 0 and 4 , separately. The CAN score 0-0.5 and 1-1.5 was considered absent and early CAN, respectively. The CAN score $\geq 2$ with or without orthostatic hypotension was considered severe and definite CAN. In this study, without confirmed CAN included absent and early CAN and with CAN included definite and severe CAN, respectively.

2.5. Statistical Analysis. Statistical analysis was applied using the SPSS 22.0 software package. Continuous variables conformed to normal distribution were shown as mean \pm standard, while continuous variables with an abnormal distribution were expressed as median (upper and lower quartiles). Categorical data was represented as number (percentage). Student's $t$-test was applied for contrast of two samples with a normal distribution. One-way ANOVA was used for comparison of multiple samples, and KruskalWallis test was used for abnormal distributions. $\chi^{2}$-test was applied for categorical variables. The Spearman analysis was applied to analyze the relationship between TIR and CART parameters. The binary logistic regression was applied to analyze the independent connection between TIR and CAN (without confirmed CAN vs. CAN) by adjusting age, diabetes duration, sex, blood pressure, lipid situation, SCr, BMI, HbAlc (\%), and other GV metrics. Furthermore, the multinomial logistic regression was applied to analyze the independent connection between TIR and different stages of CAN by adjusting age, diabetes duration, sex, blood pressure, lipid situation, SCr, BMI, HbAlc (\%), and other GV metrics. $P<0.05$ was considered statistically significant.

\section{Results}

3.1. Clinical Characteristics among Every Stage of CAN Groups. The baseline data of all individuals were exhibited in Table 1 . The mean age of all subjects was $53.11 \pm 12.86$ years, the median (upper and lower quartiles) duration of diabetes was $6[2,12]$ years. According to the CARTs, there were 228 diabetic individuals without CAN (without confirmed CAN) including absent CAN ( $n=83$ cases) and early CAN ( $n=145$ cases) and 121 diabetic individuals complicated with CAN including definite CAN ( $n=109$ cases) and severe CAN ( $n=12$ cases). The prevalence of early CAN, definite CAN, and severe CAN were $41.5 \%, 31.2 \%$, and $3.4 \%$, respectively. Compared with the absent CAN group, patients with more severe CAN showed increased levels of TG, FBG, SD, MBG, ADDR, and $M$ value $(P<0.05)$, and lower levels of TIR $(P<0.001)$. Median (upper and lower quartiles) TIR was $75.15 \mathrm{mmol} / \mathrm{L}(48.92 \mathrm{mmol} / \mathrm{L}, 90.49 \mathrm{mmol} / \mathrm{L}) 72.60 \mathrm{mmol} / \mathrm{L}$ $(52.18 \mathrm{mmol} / \mathrm{L}, 84.29 \mathrm{mmol} / \mathrm{L}), 53.23 \mathrm{mmol} / \mathrm{L}(33.24 \mathrm{mmol} / \mathrm{L}$, $69.98 \mathrm{mmol} / \mathrm{L})$, and $33.44 \mathrm{mmol} / \mathrm{L}(1.57 \mathrm{mmol} / \mathrm{L}, 75.28$ $\mathrm{mmol} / \mathrm{L}$ ) in absent CAN early CAN definite CAN, and severe CAN, respectively. In addition, the proportion of diabetic retinopathy was obviously higher with the aggravation of CAN $(P<0.05)$. However, there was no significant difference in $\mathrm{HbAlc}(\%)$ and the remedies for diabetes among different groups.
3.2. The Comparison of Clinical Characteristics by Quartiles (Q1-Q4) of TIR. Further analysis after dividing patients into groups was done according to quartiles of TIR ((Q1) $\leq 41 \%$; (Q2): 41-64\%; (Q3): 64-83\%; (Q4): >83\%). The characteristics were shown in Table 2. Firstly, patients with the highest quartiles of TIR had lower FBG, HbAlc (\%), SD, MAGE, MBG, LAGE, ADDR, and $M$ value $(P<0.001)$. Of note, for CART parameters, HR variation during position changing, Valsalva maneuver, and deep breathing all increased with ascending quartiles of TIR.

3.3. Prevalence of all Stage of CAN in Different Quartiles (Q1-Q4) of TIR. As shown in Figure 1, individuals were classified into groups according to quartiles of the TIR, the proportion of "without confirmed CAN" increased with the increase of TIR $(P<0.001)$ What else, the proportion of definite CAN decreased with the increase of TIR, while a similar decrease was found between the prevalence of severe CAN and quartiles of TIR $(P<0.05)$. (Figure 2$)$.

3.4. The Correlation of TIR and Parameters of CARTs. The correlation between TIR and CART parameters were performed with Spearman's analysis. As shown in Table 3, TIR negatively correlated with the total score of CAN $(P<0.001)$ and positively associated with heart rate variation during position changing, Valsalva maneuver, and deep breathing $(P<0.05)$.

3.5. Associations between TIR and Various Stages of CAN. Binary logistic regression was used to investigate the independent correlation between TIR and any CAN. After adjusting for age, diabetes duration, sex, blood pressure, lipid profile, SCr, BMI, and HbAlc (\%) (model 1), the data revealed that TIR (odds ratio (OR): $0.969,95 \%$ confidence interval (CI): $0.957-0.981, P<0.001)$ was obviously related to the presence of CAN. The multinomial Logistic regression still found the strong relationship between TIR and manifest or severe stage of CAN (Early CAN: $\mathrm{P}>0.05$. Manifest CAN: OR: 0.967, 95\% CI: 0.952-0.982, $\mathrm{P}<0.001$. Severe CAN: OR: 0.942, 95\% CI: $0.910-0.975, P=0.001$.) Furthermore, the association persisted after adjustment other GV metrics. However, there was no relationship between HbAlc and CAN in all models (Table 4). After categorizing TIR into quartiles, the data showed that the highest quartiles of TIR was associated with the lower presence of CAN compared with the lowest quartiles of TIR after adjusting for age, diabetes duration, sex, blood pressure, lipid profile, SCr, BMI, and HbAlc (\%) (model 1) (OR: 0.094, 95\% CI: $0.035-0.256, P<0.001$, highest vs. lowest). The correlation persisted after adjustment of other GV metrics, but the link between CAN and TIR, as a categorical, was weakened after adjusting MBG (Table 5).

\section{Discussion}

Based on our results, we found a robust association between TIR and CAN independent of HbAlc. What is more, TIR was obviously related to the presence of CAN even after adjusting for GV metrics, including SD and MAGE esc, this 
TABLE 1: The Clinical characteristics among every stage of CAN groups.

\begin{tabular}{|c|c|c|c|c|c|c|}
\hline & Absent CAN & Early CAN & Manifest CAN & Severe CAN & $\chi^{2} / t / z$ & $P$ \\
\hline$N$ & 83 & 145 & 109 & 12 & - & - \\
\hline Age (y) & $48.28 \pm 13.39$ & $52.27 \pm 12.45$ & $57.83 \pm 10.44$ & $53.92 \pm 19.80$ & 9.687 & $<0.001$ \\
\hline Diabetes duration (y) & $4.0(1.0,10.0)$ & $4.0(0.8,10.0)$ & $10.0(5.0,16.5)$ & $9.5(1.3,18)$ & 31.007 & $<0.001$ \\
\hline Male $(n, \%)$ & $(51,61.45)$ & $(105,72.41)$ & $(66,60.55)$ & $(6,50.00)$ & 6.110 & 0.106 \\
\hline Smoking $(n, \%)$ & $(33,39.76)$ & $(52,35.86)$ & $(42,38.53)$ & $(4,33.33)$ & 0.485 & 0.922 \\
\hline Retinopathy $(n, \%)$ & $(16,19.28)$ & $(33,22.76)$ & $(40,36.70)$ & $(5,41.67)$ & 10.360 & 0.016 \\
\hline SBP (mmHg) & $131.58 \pm 16.90$ & $129.83 \pm 16.06$ & $137.14 \pm 18.60$ & $135.00 \pm 19.65$ & 3.951 & 0.009 \\
\hline DBP (mmHg) & $82.19 \pm 10.85$ & $80.52 \pm 9.72$ & $79.86 \pm 10.99$ & $76.75 \pm 9.96$ & 1.379 & 0.249 \\
\hline BUN (mmol/L) & $5.06 \pm 1.31$ & $5.80 \pm 2.12$ & $6.67 \pm 2.86$ & $5.98 \pm 2.51$ & 8.158 & $<0.001$ \\
\hline $\mathrm{SCr}(\mu \mathrm{mol} / \mathrm{L})$ & $55(45,68)$ & $56(45,66)$ & $58(46,83)$ & $46(40,52)$ & 6.244 & 0.100 \\
\hline $\mathrm{TC}(\mathrm{mmol} / \mathrm{L})$ & $4.65 \pm 1.20$ & $4.72 \pm 1.23$ & $4.46 \pm 1.18$ & $5.09 \pm 1.04$ & 1.488 & 0.218 \\
\hline $\mathrm{TG}(\mathrm{mmol} / \mathrm{L})$ & $1.70(1.10,2.64)$ & $1.51(1.05,2.40)$ & $1.56(0.97,2.02)$ & $2.38(1.96,4.13)$ & 8.404 & 0.038 \\
\hline $\mathrm{HDL}(\mathrm{mmol} / \mathrm{L})$ & $0.99(0.88,1.19)$ & $1.04(0.90,1.18)$ & $1.04(0.89,1.26)$ & $1.01(0.73,1.22)$ & 1.558 & 0.669 \\
\hline $\mathrm{LDL}(\mathrm{mmol} / \mathrm{L})$ & $2.74(2.07,3.38)$ & $2.77(2.26,3.32)$ & $2.60(1.89,3.37)$ & $2.70(1.71,3.35)$ & 1.090 & 0.780 \\
\hline FBG $(\mathrm{mmol} / \mathrm{L})$ & $7.20 \pm 1.90$ & $7.24 \pm 2.71$ & $7.82 \pm 2.69$ & $11.18 \pm 3.64$ & 9.569 & $<0.001$ \\
\hline BMI & $24.87 \pm 4.00$ & $25.19 \pm 3.27$ & $25.15 \pm 3.65$ & $26.27 \pm 5.75$ & 0.540 & 0.655 \\
\hline HbA1C (\%) & $8.67 \pm 2.14$ & $9.15 \pm 2.39$ & $9.55 \pm 2.46$ & $9.88 \pm 2.04$ & 2.581 & 0.053 \\
\hline TIR (3.9-10 mmol/L) (\%) & $75.15(48.92,90.49)$ & $72.60(52.18,84.29)$ & $53.23(33.24,69.98)$ & $33.44(1.57,75.28)$ & 35.487 & $<0.001$ \\
\hline $\mathrm{SD}(\mathrm{mmol} / \mathrm{L})$ & $2.03(1.50,2.62)$ & $2.09(1.70,2.72)$ & $2.42(2.02,3.27)$ & $2.53(2.08,2.95)$ & 16.055 & 0.001 \\
\hline MAGE (mmol/L) & $1.08(0.86,1.59)$ & $1.10(0.84,1.44)$ & $1.19(0.95,1.69)$ & $1.08(0.94,1.36)$ & 5.457 & 0.141 \\
\hline MBG (mmol/L) & $9.00 \pm 1.97$ & $9.34 \pm 2.17$ & $10.38 \pm 2.36$ & $11.18 \pm 2.71$ & 9.323 & $<0.001$ \\
\hline $\mathrm{CV}$ & $0.24 \pm 0.07$ & $0.25 \pm 0.08$ & $0.26 \pm 0.12$ & $0.24 \pm 0.06$ & 1.300 & 0.274 \\
\hline LAGE (mmol/L) & $11.08(8.77,13.97)$ & $11.57(8.97,15.35)$ & $12.72(10.48,17.26)$ & $11.68(9.90,16.27)$ & 11.968 & 0.007 \\
\hline $\operatorname{ADDR}(\mathrm{mmol} / \mathrm{L})$ & $20.19(11.99,28.51)$ & $21.41(15.00,30.97)$ & $27.58(20.92,37.93)$ & $33.60(19.75,55.20)$ & 24.836 & $<0.001$ \\
\hline$M$ value $(\mathrm{mmol} / \mathrm{L})$ & $5.18(2.09,13.29)$ & $6.54(3.38,13.55)$ & $12.23(6.30,24.68)$ & $19.58(6.10,51.53)$ & 34.801 & $<0.001$ \\
\hline \multicolumn{7}{|l|}{ Treatment, $(n, \%)$} \\
\hline No treatment & $11(13.25)$ & $45(31.03)$ & $25(22.94)$ & $1(8.34)$ & 15.207 & 0.085 \\
\hline OHA & $21(25.30)$ & $38(26.21)$ & $26(23.85)$ & $4(33.33)$ & & \\
\hline Insulin & $27(32.53)$ & $31(21.38)$ & $24(22.02)$ & $3(25.00)$ & & \\
\hline OHA \& insulin & $24(28.92)$ & $31(21.38)$ & $34(31.19)$ & $4(33.33)$ & & \\
\hline
\end{tabular}

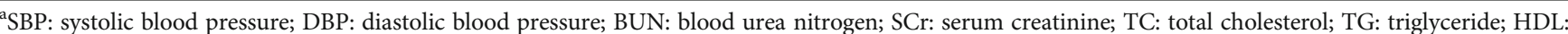
high-density lipoprotein; LDL: low-density lipoprotein; FBG: fasting blood glucose; BMI: body mass index; HbA1C: hemoglobin A1C; TIR: time in range; SD: standard deviation; MAGE: mean amplitude of glucose excursions; MBG: mean blood glucose; CV: coefficient of variation; LAGE: largest amplitude of plasma glucose excursions; ADDR: average daily risk range; OHA: oral hypoglycemic agents. ${ }^{\mathrm{b}}$ Normally distributed values in the table are presented as the means \pm SD, nonnormally distributed values are presented as medians ( $25 \%$ and $75 \%$ interquartiles), and categorical variables are presented as frequencies (percentages). ANOVA for comparison of various samples with a normal distribution. Kruskal-Wallis test for abnormal distributions. $\chi^{2}$ test for categorical variables.

result suggested that the value of TIR in predicting the risk of CAN is independent of GV metrics.

Diabetes Control and Complications Trial (DCCT) and the UK Prospective Diabetes Study (UKPDS) [11, 12] has revealed that hemoglobin $\mathrm{A} 1 \mathrm{C}$ could be used as the gold standard to assess glycemic control. The DCCT also showed the strong relationship between $\mathrm{HbAlc}$ and the danger of longterm diabetic complications. Besides, the primary goal of T2DM management is to decrease glycated HbA1c to $7 \%$ or $6.5 \%$. According to the guidelines of the American Diabetes Association, HbA1c is considered an important predictor of chronic diabetes complications [13]. The guidelines also indicated that patients with cardiovascular diseases may have a well-controlled HbAlc. However, HbAlc explained only a portion of the variation in diabetic chronic complications risk. For example, the investigators of the Diabetes Control and Complications Trial (DCCT) found that HbAlc explained only about $11 \%$ of the variation in retinopathy risk in the DCCT cohort [14]. At present, most studies demonstrated that $\mathrm{HbA} 1 \mathrm{c}$ was a risk factor for the presence and progression of CAN [15]. However, there was no relationship between $\mathrm{HbAlc}$ and CAN with adjustment for TIR in our study and there was no difference in $\mathrm{HbA} 1 \mathrm{c}$ among different stages of CAN.

Moreover, more and more evidence shows that HbAlc does have certain limitations: Firstly, HbAlc cannot provide 


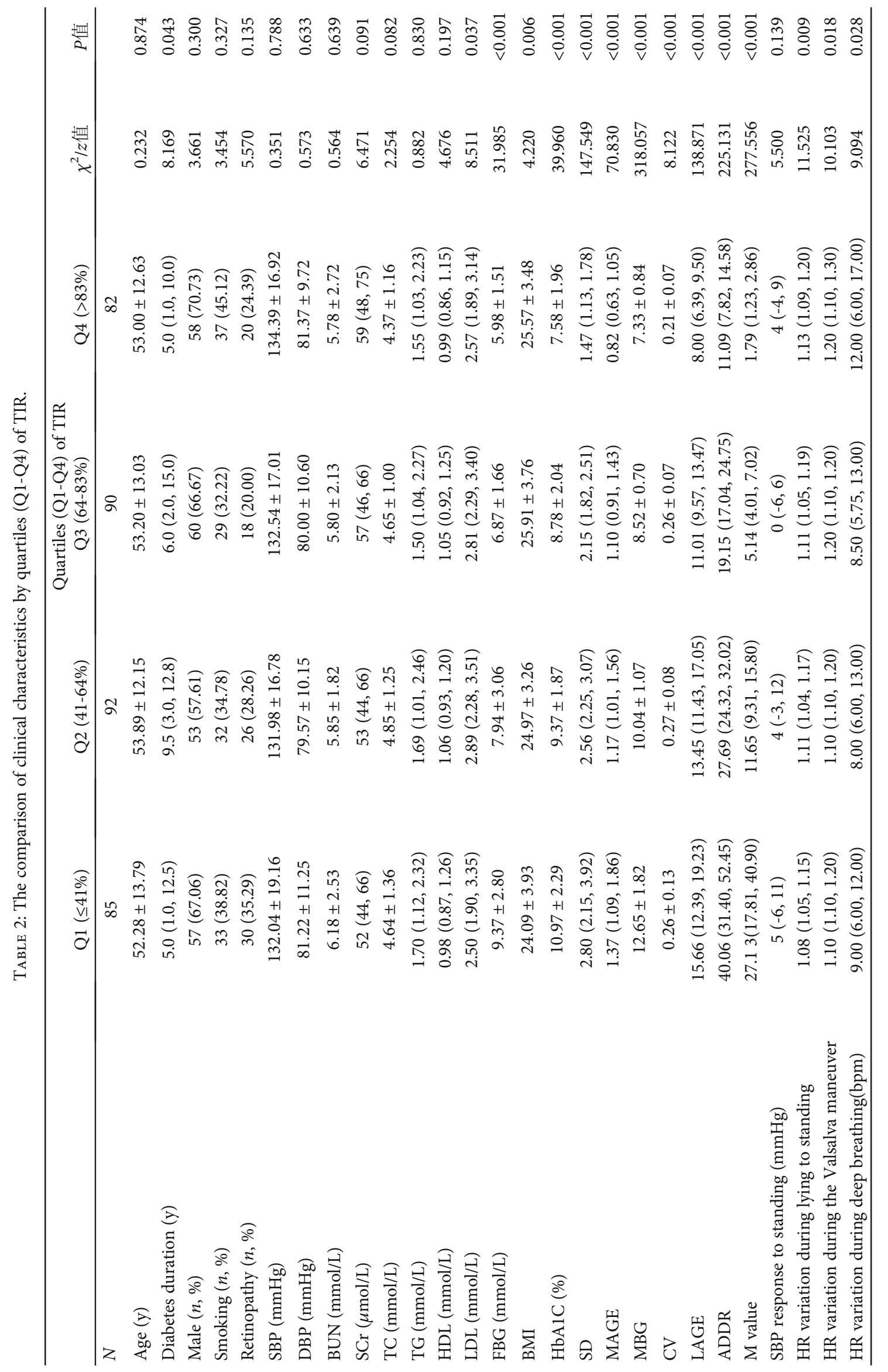




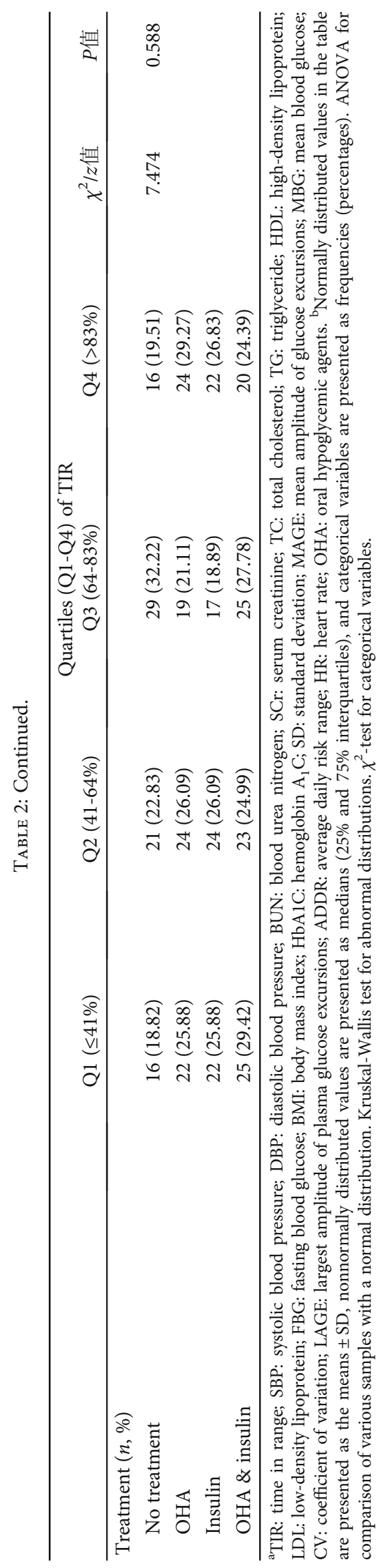




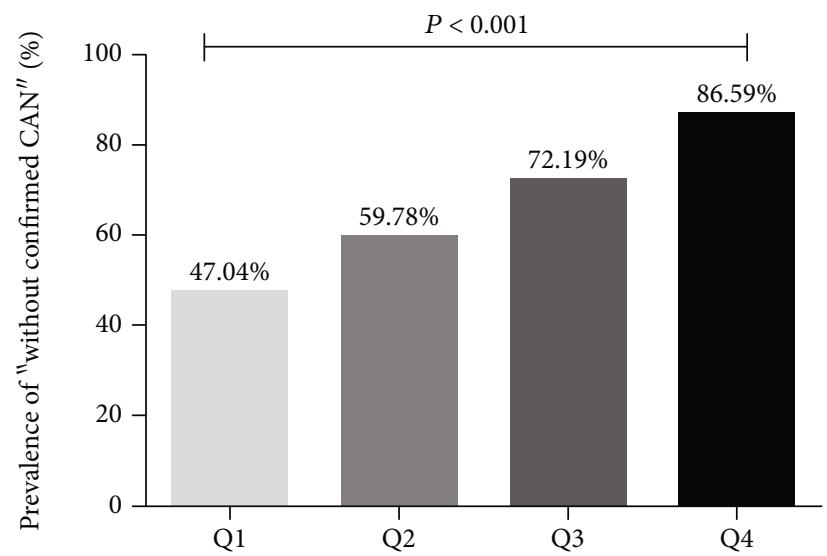

Figure 1: Prevalence of “without confirmed CAN" in different quartiles (Q1-Q4) of TIR. ${ }^{\text {a } C A N: ~ c a r d i o v a s c u l a r ~ a u t o n o m i c ~ n e u r o p a t h y . ~}{ }^{\text {b TIR }}$ Q1 $\leq 41 \%$, Q2: 41-64\%, Q3: 64-83\%, Q4 > 83\%. ${ }^{\mathrm{C}}$ As shown in this figure, patients were divided into groups according to quartiles of the time in range (TIR), the proportion of "without confirmed CAN" increased with the increase of TIR, $(P<0.001) . P$ value for the significant difference among the groups was determined by $\chi^{2}$-test.

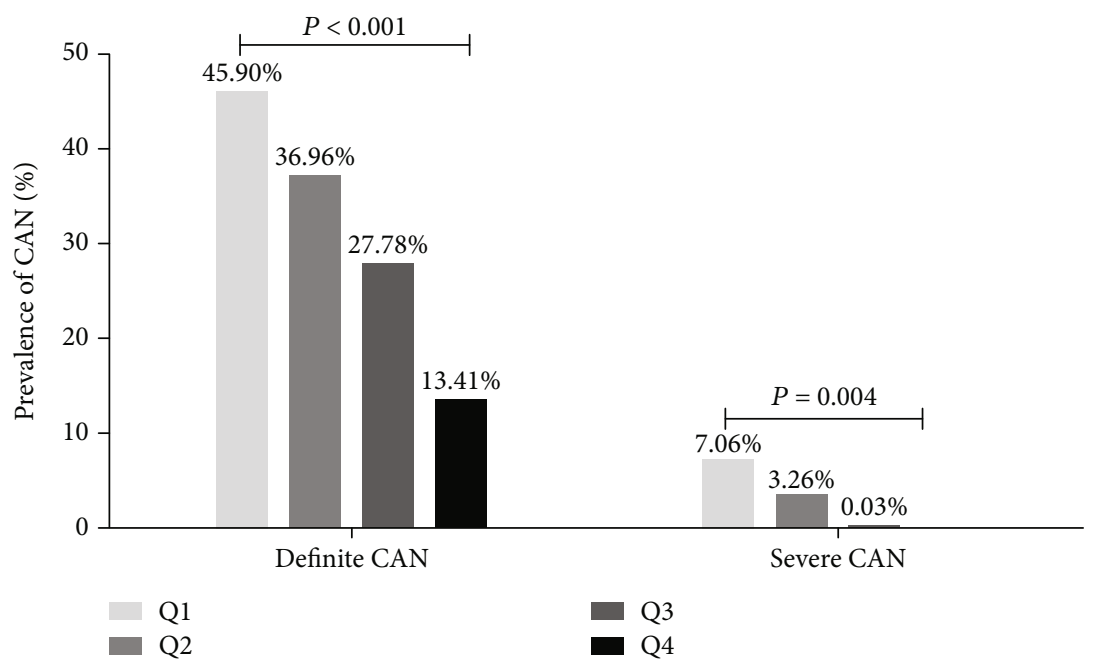

FIGURE 2: Prevalence of CAN in different quartiles (Q1-Q4) of TIR. ${ }^{a} \mathrm{CAN}$ : cardiovascular autonomic neuropathy; ${ }^{\mathrm{b}} \mathrm{TIR}$ Q1 $\leq 41 \%$, Q2: 41-64\%, Q3: 64-83\%, Q4 > 83\%. ${ }^{\mathrm{c}}$ As shown in this figure, patients were divided into groups according to quartiles of the time in range (TIR), the proportion of definite CAN decreased with the increase of TIR, while a similar decreased was found between the prevalence of severe CAN and quartiles of TIR $(P<0.05)$. $P$ value for the significant difference among the groups was determined by $\chi^{2}$-test.

TABLE 3: The correlation of TIR and parameters of CARTs.

\begin{tabular}{lcccccc}
\hline & $\begin{array}{c}\text { SBP response to } \\
\text { standing }(\mathrm{mmHg})\end{array}$ & $\begin{array}{c}\text { HR variation during } \\
\text { lying to standing }\end{array}$ & $\begin{array}{c}\text { HR variation during the } \\
\text { Valsalva maneuver }\end{array}$ & $\begin{array}{c}\text { HR variation during } \\
\text { deep breathing }(\mathrm{bpm})\end{array}$ & Total score of CAN \\
\hline \multirow{2}{*}{ TIR (3.9-10 mmol/L) } & $R$ & -0.055 & 0.167 & 0.139 & 0.121 & -0.261 \\
& $P$ & 0.302 & 0.002 & 0.010 & 0.024 & $<0.001$ \\
\hline
\end{tabular}

TIR: time in range; CARTs: cardiac autonomic reflex tests; SBP: systolic blood pressure; HR: heart rate.

information on daily hypoglycemia or hyperglycemia and daily patterns of glycemia. Secondly, the same HbAlc may correspond to a different TIR value [16]. Finally, the accuracy of its measurement is affected by a variety of clinical conditions such as hemoglobinopathies, anemia, uremia, and pregnancy [17].
TIR correlates highly with HbAlc, suggesting that TIR may be used as a novel and promising metric in assessing the risk of diabetes complications and glycemic status in diabetic patients. Compared with $\mathrm{HbAlc}$ testing, TIR provides more sensitive and accurate results. As an example, TIR assessment can record acute events of hypoglycemia or 


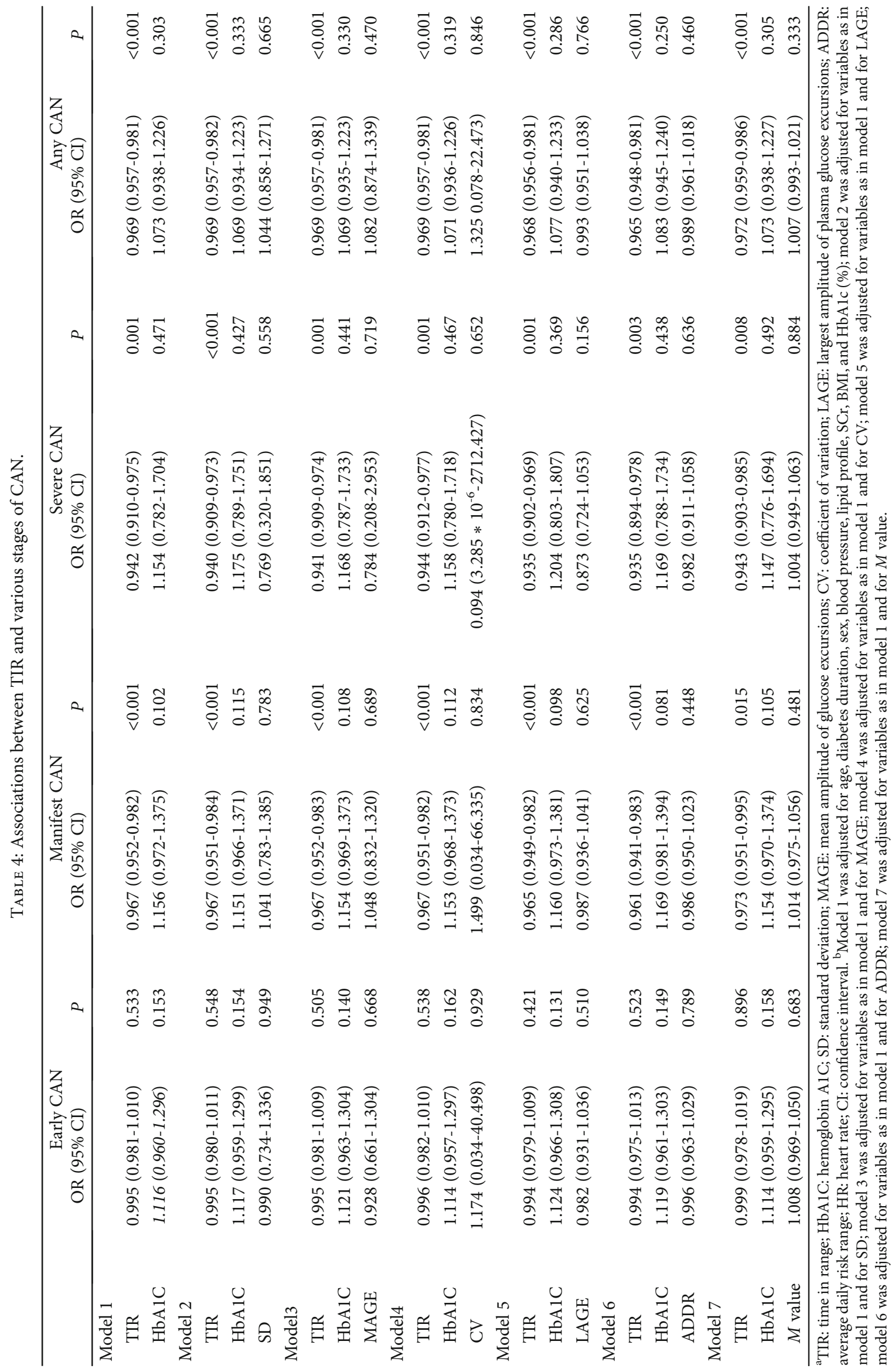


TABLE 5: Association between quartiles of TIR and CAN.

\begin{tabular}{|c|c|c|c|c|c|}
\hline & $\beta$ & S.E. & Wald & $P$ & OR (95\% CI) \\
\hline \multicolumn{6}{|l|}{ Model 1} \\
\hline TIR Q1 & - & - & - & - & 1 (reference) \\
\hline TIR Q2 & -0.686 & 0.367 & 3.483 & 0.062 & $0.504(0.245-1.035)$ \\
\hline TIR Q3 & -1.265 & 0.393 & 10.347 & 0.001 & $0.282(0.131-0.610)$ \\
\hline TIR Q4 & -2.364 & 0.511 & 21.394 & $<0.001$ & $0.094(0.035-0.256)$ \\
\hline \multicolumn{6}{|l|}{ Model 2} \\
\hline TIR Q1 & - & - & - & - & 1 (reference) \\
\hline TIR Q2 & -0.676 & 0.371 & 3.332 & 0.068 & $0.508(0.246-1.051)$ \\
\hline TIR Q3 & -1.250 & 0.403 & 9.637 & 0.002 & $0.287(0.130-0.631)$ \\
\hline TIR Q4 & -2.337 & 0.532 & 19.307 & $<0.001$ & $0.097(0.034-0.274)$ \\
\hline SD & 0.018 & 0.099 & 0.033 & 0.856 & $1.018(0.838-1.237)$ \\
\hline \multicolumn{6}{|l|}{ Model 3} \\
\hline TIR Q1 & - & - & - & - & 1 (reference) \\
\hline TIR Q2 & -0.649 & 0.370 & 3.084 & 0.079 & $0.523(0.253-1.078)$ \\
\hline TIR Q3 & -1.229 & 0.395 & 9.663 & 0.002 & $0.292(0.135-0.635)$ \\
\hline TIR Q4 & -2.312 & 0.514 & 20.241 & $<0.001$ & $0.099(0.036-0.271)$ \\
\hline MAGE & 0.071 & 0.110 & 0.419 & 0.517 & $1.074(0.866-1.331)$ \\
\hline \multicolumn{6}{|l|}{ Model 4} \\
\hline TIR Q1 & - & - & - & - & 1 (reference) \\
\hline TIR Q2 & -0.675 & 0.369 & 3.357 & 0.067 & $0.509(0.247-1.048)$ \\
\hline TIR Q3 & -1.254 & 0.394 & 10.120 & 0.001 & $0.285(0.132-0.618)$ \\
\hline TIR Q4 & -2.387 & 0.514 & 21.538 & $<0.001$ & $0.092(0.034-0.252)$ \\
\hline $\mathrm{CV}$ & -0.646 & 1.471 & 0.193 & 0.661 & $0.524(0.029-9.372)$ \\
\hline \multicolumn{6}{|l|}{ Model 5} \\
\hline TIR Q1 & - & - & - & - & 1 (reference) \\
\hline TIR Q2 & -0.701 & 0.370 & 3.598 & 0.058 & $0.496(0.240-1.024)$ \\
\hline TIR Q3 & -1.303 & 0.405 & 10.358 & 0.001 & $0.272(0.123-0.601)$ \\
\hline TIR Q4 & -2.425 & 0.534 & 20.615 & $<0.001$ & $0.088(0.031-0.252)$ \\
\hline LAGE & -0.009 & 0.022 & 0.162 & 0.688 & $0.991(0.948-1.036)$ \\
\hline \multicolumn{6}{|l|}{ Model 6} \\
\hline TIR Q1 & - & - & - & - & 1 (reference) \\
\hline TIR Q2 & -0.720 & 0.404 & 3.169 & 0.075 & $0.487(0.221-1.075)$ \\
\hline TIR Q3 & -1.319 & 0.474 & 7.750 & 0.005 & $0.267(0.106-0.677)$ \\
\hline TIR Q4 & -2.439 & 0.631 & 14.916 & $<0.001$ & $0.087(0.025-0.301)$ \\
\hline ADDR & -0.003 & 0.014 & 0.041 & 0.839 & $0.997(0.971-1.025)$ \\
\hline \multicolumn{6}{|l|}{ Model 7} \\
\hline TIR Q1 & - & - & - & - & 1 (reference) \\
\hline TIR Q2 & -0.417 & 0.426 & 0.957 & 0.328 & $0.659(0.286-1.519)$ \\
\hline TIR Q3 & -0.917 & 0.487 & 3.541 & 0.060 & $0.400(0.154-1.039)$ \\
\hline TIR Q4 & -1.958 & 0.602 & 10.583 & 0.001 & $0.141(0.043-0.459)$ \\
\hline$M$ value & 0.015 & 0.013 & 1.306 & 0.253 & $1.015(0.990-1.040)$ \\
\hline
\end{tabular}

${ }^{\mathrm{a} T I R: ~ t i m e ~ i n ~ r a n g e ; ~ S D: ~ s t a n d a r d ~ d e v i a t i o n ; ~ M A G E: ~ m e a n ~ a m p l i t u d e ~ o f ~ g l u c o s e ~ e x c u r s i o n s ; ~ C V: ~ c o e f f i c i e n t ~ o f ~ v a r i a t i o n ; ~ L A G E: ~ l a r g e s t ~ a m p l i t u d e ~ o f ~ p l a s m a ~}$ glucose excursions; ADDR: average daily risk range; HR: heart rate; CI: confidence interval. ${ }^{\text {b }}$ Model 1 was adjusted for age, diabetes duration, sex, blood pressure, lipid profile, SCr, BMI, and HbAlc (\%); model 2 was adjusted for variables as in model 1 and for SD; model 3 was adjusted for variables as in model 1 and for MAGE; model 4 was adjusted for variables as in model 1 and for CV; model 5 was adjusted for variables as in model 1 and for LAGE; model 6 was adjusted for variables as in model 1 and for ADDR; model 7 was adjusted for variables as in model 1 and for $M$ value. ${ }^{\mathrm{C}}$ TIR $Q 1 \leq 41 \%$, Q2: $41-64 \%$, Q3: 64-83\%,Q4 > 83\%.

hyperglycemia at any time. Obviously, this data cannot be obtained in HbAlc assessment [18]. Omar esc demonstrated that individuals with lower than $80 \%$ TIR had worse clinical outcomes than those with higher than $80 \%$ TIR, regardless if they had diabetes or not [19]. Recent trials revealed that TIR and $\mathrm{HbAlc}$ had similar correlation with chronic diabetic 
complications. Beck et al. found a strong relationship between TIR and microvascular complications, including diabetic microalbuminuria and retinopathy. The authors also indicated that patients with complications had a decreased TIR (10-12\%) in contrast with those who did not. With each $10 \%$ decrease in TIR, the risk of retinopathy and microalbuminuria raised by $64 \%$ and $40 \%$, separately [9]. Lu et al. explored the relationship between TIR measured by CGM and retinopathy in T2DM. The data showed that individuals with higher TIR had lower risk of developed DR. In addition, TIR had an HbA1c-independent relationship with the prevalence of DR. The association between TIR and the presence of all stages of DR remained unchanged after adjusting GV metrics. This data firstly found the significant effect of TIR on DR independent of GV metrics [20]. A small sample (80 cases) clinical study reported that CAN was negatively associated with percent time in glucose ranging from 70 to $180 \mathrm{mg} / \mathrm{dL}$ in type 1 diabetes [21].

A great number of previous studies found the glucoseindependent correlation between GV and CAN in newly diagnosed type 2 diabetes. In these study, metrics assessed by CGM such as SD and MAGE esc provided more information beyond $\mathrm{HbAlc}$. Considering that $\mathrm{HbAlc}$ had no great difference between CAN and without confirmed CAN group, monitoring glucose patterns more than 24 hours may play a more important role than $\mathrm{HbA} 1 \mathrm{c}$ on glucose management in individuals with T2DM and CAN [22-24]. In concert with another study, our results showed that there was no obvious difference in $\mathrm{HbAlc}(\%)$ among different groups. Compared with absent CAN group, patients with more severe CAN showed increased levels of SD, MBG, ADDR, and $M$ value $(P<0.05)$. Logistic regression also revealed TIR was reversely associated with the presence of CAN even after adjusting for GV metrics and HbAlc (\%); these results suggested that the value of TIR in predicting the risk of CAN is independent of GV metrics and HbAlc (\%).

Short-term hypoglycemia, hyperglycemia, and glycemic fluctuations had an important role in the development of CAN. CAN is the result of the combined action of multiple factors, and its pathogenesis is mainly related to the metabolic disorder, vascular injury, inflammatory reaction, and oxidative stress [25]. The molecular mechanisms related to the association between TIR and CAN have not been clarified yet, which may be the role of oxidative stress. Previous animal and in vitro studies have indicated a robust association between glycemic fluctuations, hypoglycemia, and oxidative stress previously [26], which was also confirmed in several human studies [27].

However, the present study has several limitations. Firstly, considering the small overall sample size in our study, a multicenter larger sample size is needed to confirm the relationship between TIR and CAN. Secondly, we did not conduct the prospective study, making it impossible to investigate the causal relationship between TIR and CAN.

\section{Conclusion}

In conclusion, our work revealed that TIR is associated with the presence of any stages for CAN independent of HbAlc and GV metrics. TIR and other glycemic parameters assessed by CGM have high values and development potential as outcome measures.

\section{Data Availability}

The datasets generated during and/or analyzed during the current study are available from the corresponding authors and the senior author on reasonable request.

\section{Ethical Approval}

This study was approved by the Ethics Committee of Jinling Hospital, Nanjing University and was performed according to the Declaration of Helsinki.

\section{Conflicts of Interest}

The authors declare that they have no conflict of interest.

\section{Authors' Contributions}

Qingyu Guo, Pu Zang, Shaoying Xu, Zhen Zhang, and Chunyan Liu conceived and designed the research. Qingyu Guo, Wenjing Song, and Zhanhong Guo analyzed and interpreted the data. Qingyu Guo, Jing Chen, and Bin Lu performed the statistical analysis. Qingyu Guo wrote the manuscript. Ping Gu and Jiaqing Shao critically revised the manuscript for key intellectual content. Qingyu Guo is responsible for the integrity of the work. All authors read and approved the final manuscript. Qingyu Guo, Pu Zang, and Shaoying $\mathrm{Xu}$ contributed equally to this paper.

\section{Acknowledgments}

This work was supported by the National Natural Science Foundation of China (81774134, 81873174), Natural Science Foundation of Jiangsu Province of China (BK20171331, BK20150558), Postdoctoral Foundation of Jiangsu Province of China (1501120C), and Jiangsu Province 333 Talent Funding Project (BRA2017595).

\section{References}

[1] Y. M. Luijf, A. Avogaro, C. Benesch et al., "Continuous glucose monitoring accuracy results vary between assessment at home and assessment at the clinical research center," Journal of Diabetes Science and Technology, vol. 6, no. 5, pp. 1103-1106, 2012.

[2] T. Danne, R. Nimri, T. Battelino et al., "International consensus on use of continuous glucose monitoring," Diabetes Care, vol. 40, no. 12, pp. 1631-1640, 2017.

[3] Beyond A1C Writing Group, "Need for regulatory change to incorporate beyond A1C glycemic metrics," Diabetes Care, vol. 41, no. 6, pp. e92-e94, 2018.

[4] M. R. Rickels, A. J. Peleckis, E. Markmann et al., "Long-term improvement in glucose control and counterregulation by islet transplantation for type 1 diabetes," The Journal of Clinical Endocrinology and Metabolism, vol. 101, no. 11, pp. 44214430, 2016. 
[5] J. Gerritsen, J. M. Dekker, B. J. Ten Voorde et al., "Impaired autonomic function is associated with increased mortality, especially in subjects with diabetes, hypertension, or a history of cardiovascular disease: the Hoorn Study," Diabetes Care, vol. 24, no. 10, pp. 1793-1798, 2001.

[6] R. Pop-Busui, G. W. Evans, H. C. Gerstein et al., "Effects of cardiac autonomic dysfunction on mortality risk in the Action to Control Cardiovascular Risk in Diabetes (ACCORD) trial," Diabetes Care, vol. 33, no. 7, pp. 1578-1584, 2010.

[7] J. Fleischer, "Diabetic autonomic imbalance and glycemic variability," Journal of Diabetes Science and Technology, vol. 6, no. 5, pp. 1207-1215, 2012.

[8] G. Su, S. Mi, H. Tao et al., "Association of glycemic variability and the presence and severity of coronary artery disease in patients with type 2 diabetes," Cardiovascular Diabetology, vol. 10, article 19, 2011.

[9] R. W. Beck, R. M. Bergenstal, T. D. Riddlesworth et al., "Validation of time in range as an outcome measure for diabetes clinical trials," Diabetes Care, vol. 42, no. 3, pp. 400-405, 2019.

[10] V. Spallone, F. Bellavere, L. Scionti et al., "Recommendations for the use of cardiovascular tests in diagnosing diabetic autonomic neuropathy," Nutrition, Metabolism, \& Cardiovascular Diseases, vol. 21, no. 1, pp. 69-78, 2011.

[11] The DCCT Research Group, "The Diabetes Control and Complications Trial (DCCT). Design and methodologic considerations for the feasibility phase. The DCCT Research Group," Diabetes, vol. 35, no. 5, pp. 530-545, 1986.

[12] UK Prospective Diabetes Study (UKPDS) Group, "Intensive blood-glucose control with sulphonylureas or insulin compared with conventional treatment and risk of complications in patients with type 2 diabetes (UKPDS 33)," The Lancet, vol. 352, no. 9131, pp. 837-853, 1998.

[13] American Diabetes Association, "Standards of medicalcare in diabetes-2017: summary of revisions," Diabetes Care, vol. 39, Supplement 1, pp. S4-S5, 2017.

[14] J. M. Lachin, S. Genuth, D. M. Nathan, B. Zinman, B. N. Rutledge, and DCCT/EDIC Research Group, "Effect of glycemic exposure on the risk of microvascular complications in the diabetes control and complications trial-revisited," Diabetes, vol. 57, no. 4, pp. 995-1001, 2008.

[15] S. T. Andersen, D. R. Witte, J. Fleischer et al., "Risk factors for the presence and progression of cardiovascular autonomic neuropathy in type 2 diabetes: ADDITION-Denmark," Diabetes Care, vol. 41, no. 12, pp. 2586-2594, 2018.

[16] R. A. Vigersky and C. McMahon, "The relationship of hemoglobin A1C to time-in-range in patients with diabetes," Diabetes Technology \& Therapeutics, vol. 21, no. 2, pp. 81-85, 2019.

[17] L. A. Wright and I. B. Hirsch, "Metrics beyond hemoglobin A1C in diabetes management: time in range, hypoglycemia, and other parameters," Diabetes Technology \& Therapeutics, vol. 19, no. S2, pp. S16-S26, 2017.

[18] R. M. Cohen, Y. R. Holmes, T. C. Chenier, and C. H. Joiner, "Discordance between HbAlc and fructosamine: evidence for a glycosylation gap and its relation to diabetic nephropathy," Diabetes Care, vol. 26, no. 1, pp. 163-167, 2003.

[19] A. S. Omar, A. Salama, M. Allam et al., "Association of time in blood glucose range with outcomes following cardiac surgery," BMC Anesthesiology, vol. 15, article 14, 2015.

[20] J. Lu, X. Ma, J. Zhou et al., "Association of time in range, as assessed by continuous glucose monitoring, with diabetic retinopathy in type 2 diabetes," Diabetes Care, vol. 41, no. 11, pp. 2370-2376, 2018.

[21] J. E. Jun, S. E. Lee, Y. B. Lee et al., "Continuous glucose monitoring defined glucose variability is associated with cardiovascular autonomic neuropathy in type 1 diabetes," Diabetes/Metabolism Research and Reviews, vol. 35, no. 2, article e3092, 2019.

[22] J. E. Jun, S. M. Jin, J. Baek et al., "The association between glycemic variability and diabetic cardiovascular autonomic neuropathy in patients with type 2 diabetes," Cardiovascular Diabetology, vol. 14, article 70, 2015.

[23] D. Matsutani, M. Sakamoto, H. Iuchi et al., "Glycemic variability in continuous glucose monitoring is inversely associated with baroreflex sensitivity in type 2 diabetes: a preliminary report," Cardiovascular Diabetology, vol. 17, no. 1, p. 36, 2018.

[24] W. Xu, Y. Zhu, X. Yang et al., "Glycemic variability is an important risk factor for cardiovascular autonomic neuropathy in newly diagnosed type 2 diabetic patients," International Journal of Cardiology, vol. 215, pp. 263-268, 2016.

[25] M. Kuehl and M. J. Stevens, "Cardiovascular autonomic neuropathies as complications of diabetes mellitus," Nature Reviews Endocrinology, vol. 8, no. 7, pp. 405-416, 2012.

[26] E. M. Horváth, R. Benkő, L. Kiss et al., "Rapid 'glycaemic swings' induce nitrosative stress, activate poly(ADP-ribose) polymerase and impair endothelial function in a rat model of diabetes mellitus," Diabetologia, vol. 52, no. 5, pp. 952-961, 2009.

[27] I. M. Wentholt, W. Kulik, R. P. Michels, J. B. Hoekstra, and J. DeVries, "Glucose fluctuations and activation of oxidative stress in patients with type 1 diabetes," Diabetologia, vol. 51, no. 1, pp. 183-190, 2008. 